livraisons

d'Histoire

de l'Architecture

\section{Livraisons de l'histoire de l'architecture}

$20 \mid 2010$

Ceci tuera cela

\title{
« Ceci tuera cela » à l'écran
}

"This will kill that » for the cinema

«Dieses wird jenes töten » im Film

\section{Arnaud Laster}

\section{OpenEdition}

\section{Journals}

Édition électronique

URL : http://journals.openedition.org/lha/255

DOI : 10.4000/lha.255

ISSN : 1960-5994

Éditeur

Association Livraisons d'histoire de l'architecture - LHA

Édition imprimée

Date de publication : 10 décembre 2010

Pagination : $35-48$

ISSN : $1627-4970$

\section{Référence électronique}

Arnaud Laster, « "Ceci tuera cela » à l'écran », Livraisons de l'histoire de l'architecture [En ligne], 20 |

2010, mis en ligne le 10 décembre 2012, consulté le 06 février 2020. URL : http://

journals.openedition.org//ha/255; DOI : 10.4000/lha.255

Ce document a été généré automatiquement le 6 février 2020

Tous droits réservés à l'Association LHA 


\title{
«Ceci tuera cela » à l'écran
}

\author{
"This will kill that " for the cinema \\ «Dieses wird jenes töten » im Film
}

Arnaud Laster

1 La consultation de l'archidiacre Claude Frollo par Louis XI à Notre-Dame et le chapitre "Ceci tuera cela » sont si peu nécessaires à l'intrigue de Notre-Dame de Paris qu'ils ne figuraient pas, c'est bien connu, dans les premières éditions du roman de Victor Hugo. Le cinéma ayant coutume de ne conserver des romans qu'il transpose que la seule trame, on aurait pu s'attendre à ne pas voir restitués à l'écran cet épisode ni surtout la vaste réflexion qu'il suscite chez le narrateur. Or l'un et l'autre se retrouvent partiellement dans deux films au moins parmi les très nombreux qui ont été adaptés de Notre-Dame de Paris ${ }^{1}$ : la version réalisée aux États-Unis en 1939 par William Dieterle sous le titre The Hunchback of Notre-Dame (Le Bossu de Notre-Dame) ${ }^{2}$ - diffusée, l'année suivante, en France sous le titre Quasimodo - et le téléfilm réalisé par Peter Medak, intitulé The Hunchback ${ }^{3}$ - diffusé pour la première fois en mars 1997 par la chaîne du câble TNT (Turner Network Television) et ultérieurement en France sous le titre Quasimodo, Notre-Dame de Paris. Mais la source hugolienne a été assez profondément transformée et ce sont ces remaniements sur lesquels je voudrais appeler l'attention pour en conjecturer les causes et les finalités.

\section{La version de Dieterle : antiraciste mais soumise au code Hays}

2 Le carton initial du film de Dieterle, censé présenter le contexte historique de l'intrigue, a de quoi étonner un lecteur de Notre-Dame de Paris : « Le peuple sous Louis XI se sentit libre d'espérer de nouveau - un rêve de progrès. Mais superstition et préjugés souvent se dressaient en travers du chemin, cherchant à étouffer l'esprit aventureux de l'homme ${ }^{4}$.» Ce texte que l'on pourrait supposer à destination du public américain se retrouve à peu de choses près dans la version française du film : "Sous le règne de Louis XI, le peuple se reprit à espérer en une période plus heureuse. Mais la 
superstition sévissait encore... » Voilà qui annonce la réhabilitation d'un roi dont le roman de Hugo donne un portrait peu flatteur. Serait-ce une première application du code de production des films, connu sous le nom de code Hays, en vigueur aux ÉtatsUnis de 1934 à 1966, qui prescrit que "l'histoire, les institutions, les personnages importants et les citoyens d'autres nations seront présentés avec impartialité ${ }^{\prime}$ »? Mais, puisque ce même code Hays édicte que «les ministres du culte en tant que tels ne devraient pas être utilisés en tant que personnages comiques ou méchants ", comment les adaptateurs, Bruno Frank et Sonya Levien, ont-ils bien pu s'y prendre pour représenter « superstition et préjugés » autrement que par l'archidiacre Claude Frollo, et quels personnages du roman incarnent alors « le peuple » rêvant de "progrès »?

3 Le prologue du film emprunte sa substance aux chapitres 1 et 2 du livre $V$; il transpose donc à sa manière le "Ceci tuera cela", sans que la formule soit pour autant prononcée. Mais, tenus de se conformer aux impératifs du code Hays, les scénaristes de Dieterle ont changé l'archidiacre Claude Frollo en un "Grand Juge » qui hérite du prénom de son jeune frère dans le roman, Jehan ; on découvrira plus tard que ce Grand Juge a lui aussi un frère, en la personne d'un archevêque exemplaire, prénommé Claude $^{6}$. Le lieu de la scène n'est plus, comme chez Hugo, la cellule de chanoine de l'archidiacre, mais une imprimerie. Au médecin du roi, Jacques Coictier, a été substitué un imprimeur, maître Fisher. Il ne s'agit pas d'une consultation médicale à l'initiative d'un Louis XI incognito, qui dérive vers une discussion philosophique, mais d'une visite organisée par un haut magistrat désireux d'attirer l'attention du souverain sur une machine nouvelle dont maître Fisher se hâte de nommer l'inventeur, Gutenberg, d'énoncer l'utilité - faciliter au peuple l'apprentissage de la lecture -, et de vanter le caractère économique. Frollo, lui, en est horrifié et use, pour expliquer son effroi, des mêmes termes que son modèle dans le roman : "Les petites choses viennent à bout des grandes [...]. Le rat du Nil tue le crocodile ${ }^{7}$.» Mais au lieu de prononcer là des «paroles énigmatiques ", d'exprimer " une pensée qui se dérobait ${ }^{8}$ " à ses interlocuteurs et que le narrateur se chargera d'expliciter, il se fait parfaitement comprendre d'eux. "Cette petite presse à imprimer peut, déclare-t-il, détruire un royaume.» Ce sont donc les conséquences politiques possibles de l'invention qu'il met d'abord en avant, alors que le romancier les mentionnait comme la traduction rationnelle de la prophétie contenue dans "Ceci tuera cela", le "pronostic du philosophe qui voit la pensée humaine, volatilisée par la presse, s'évaporer du récipient théocratique ", pronostic complété, neuf paragraphes plus loin, par cette «loi»: «Toute civilisation commence par la théocratie et finit par la démocratie ${ }^{9}$.» Cependant, le Louis XI de Dieterle ${ }^{10}$ ne prend pas la menace au sérieux, la trouvant " exagérée ». Ferme-t-il pour autant la porte à un traitement politique de la formule par les adaptateurs? La piste va-t-elle être abandonnée?

4 Voici que l'imprimeur sort de la presse un feuillet dont le roi se montre curieux. C'est la première page d'un nouveau livre intitulé De la liberté de pensée. Bien différent était l'infolio imprimé que le Frollo du roman feuilletait avant de recevoir la visite royale dont les adaptateurs ont fait le prologue du film : « le seul produit de la presse que renfermât sa cellule ${ }^{11}$ » était un Commentaire sur les épîtres de saint Paul (Glossa in epistolas D. Pauli ${ }^{12}$ ). On pourrait imaginer que le titre de l'ouvrage du film a été inspiré aux adaptateurs par celui du livre sur lequel Frollo nous était montré par Hugo, le coude appuyé : De Praedestinatione et libero Arbitrio ${ }^{13}$. Mais du libre arbitre à la liberté de pensée il y a plus qu'une nuance, et le volume du théologien du XII siècle ne sert que d'accoudoir tandis 
que le livre imprimé du film est présenté comme l'œuvre d'un contemporain de l'action, Pierre Gringoire, auquel les adaptateurs vont attribuer un rôle déterminant dans l'intrigue. Remarquons que, contre toute vraisemblance, le titre ne fait pas sourciller le roi, alors que Frollo en dénonce aussitôt l'auteur : «Un hérétique, sire; le diffuser, c'est communiquer la maladie.» Le vocabulaire employé par Frollo est peutêtre, de la part des scénaristes, une façon de contourner le code Hays et de suggérer l'origine ecclésiastique du personnage. Sa figure d'homme ascétique et solitaire et son rattachement spatial à l'église iront dans le même sens. Quoi qu'il en soit, la réaction de Frollo est dans le droit fil du premier sens attribué par Hugo au «Ceci tuera cela » du prêtre : «La presse tuera l'église ${ }^{14}$.»

«Cela peut être une grande bénédiction pour la France, réplique le roi, si le peuple est en mesure de se procurer des livres et d'apprendre à lire. Pour moi, c'est une nouvelle forme d'expression de la pensée. Là dehors est l'ancienne; partout en France, dans chaque cité, se dressent des cathédrales comme celle-ci, triomphants monuments du passé [...], tels de puissants gardiens, conservant vivante la foi invincible des chrétiens. Chaque arche, chaque colonne, chaque statue est un feuillet taillé dans notre histoire, glorifiant l'esprit de la France. »

Ces paroles sont en quelque sorte illustrées par des plans de coupe de Notre-Dame et de ses statues. "Les cathédrales sont l'écriture du passé. La presse est celle de notre temps. Et je ne ferai rien pour l'arrêter, Frollo ", conclut Louis XI qui, dans le roman, se contentait d'écouter et de questionner incognito. Superficiellement, on pourrait croire que l'on a simplement glissé du premier sens attribué par Hugo à « ceci tuera cela » au second, « l'imprimerie tuera l'architecture », abondamment glosé par le narrateur dans des termes voisins de ceux du Louis XI de Dieterle : «Jusqu'à Guttemberg, l'architecture est l'écriture principale, l'écriture universelle. [...] Il faut relire le passé sur ces tables de marbre. Il faut admirer et refeuilleter sans cesse le livre écrit par l'architecture ; mais il ne faut pas nier la grandeur de l'édifice qu'élève à son tour l'imprimerie ${ }^{15}$. " En fait, trois détournements majeurs ont été opérés : le commentaire du narrateur a été prêté au roi ; les cathédrales sont devenues, dans les propos du monarque, le conservatoire de l'esprit national - idée étrangère à Hugo - et d'un christianisme indépassable fonction assignée par le narrateur plutôt aux édifices de style roman, "emblème inaltérable du catholicisme pur $^{16}$ ", qu'aux cathédrales gothiques, œuvres d'un art présenté par Hugo comme plus libre et plus populaire, ouvert à la fantaisie et au caprice; enfin et surtout, le passage se fait en douceur: pas d'affrontement entre les deux médias; l'un ne tuera pas l'autre mais lui succédera et, si l'on s'en tient aux propos du roi, lui a déjà succédé.

6 Pourtant, là où Louis XI discerne un mode de diffusion moderne de la pensée, auquel il refuse de s'opposer, Frollo, dans la logique de ses rejets précédents, voit une menace de destruction pour ce qu'il appelle "notre ordre ancien et sacré ", et il annonce sa décision de protéger la France des livres imprimés comme des sorcières, des magiciens et des Gitans, "cette race étrangère qui est en train d'envahir toute l'Europe ». À l'évidence, dans le contexte de 1939, le cinéaste, originaire d'Allemagne ${ }^{17}$ - où les nazis ont brûlé des livres dès leur arrivée au pouvoir en 1933 - et Bruno Frank, qui en arrive et a écrit le premier tiers de l'adaptation en allemand ${ }^{18}$, ont décidé de faire de Frollo le porte-parole et l'agent principal de l'obscurantisme, de la xénophobie et du racisme ; une politique qui est celle de Hitler mais aussi, aux États-Unis, d'individus et de clans protectionnistes, nostalgiques de la ségrégation et hostiles à l'immigration. Le prologue met ainsi en place, autour du livre imprimé de Gringoire, symboliquement consacré à la 
défense de la liberté de pensée, les éléments d'un conflit, non entre l'église et la presse ni entre la théocratie et la démocratie, mais entre deux orientations idéologiques : l'une incarnée par Frollo et lourde du poids des préjugés et des superstitions, l'autre progressiste et encouragée par un monarque bienveillant et libéral. Qui l'emportera, de celle-ci ou de celle-là, non plus dans l'avenir mais ici et maintenant?

7 La monarchie est d'autant moins mise en question que le roi va se voir confirmé presque tout au long du film comme l'exemple du souverain éclairé; il est même tenté d'admettre que la terre est ronde et de financer Christophe Colomb, contre l'avis de Frollo. Il n'accepte pas les aveux obtenus par la torture, ne partage pas les préjugés hostiles aux Gitans ni les superstitions de son temps, à l'exception de celle qui consiste à accorder à l'ordalie le pouvoir d'établir l'innocence ou la culpabilité de la personne accusée (en l'occurrence Esmeralda). Aux opinions avancées du roi et aux termes par lesquels il refuse, dans le prologue, de briser la presse à imprimer et de pendre l'imprimeur comme le demandait Frollo - «Je ne suis pas aussi fou que ça » ("I'm not such $a$ fool ») - semblent faire écho ceux du récitant de la pièce, que le roi invite à écouter et qui n'est autre que Gringoire ${ }^{19}$ : «L'ancien ne peut jamais durer!/ Le nouveau réclame sa place. / C'est folie que de s'accrocher au passé. » («It's foolish to cling to the past. ») La leçon de la pantomime qui suit, «Prends garde de ne point vivre en vain ", sorte de variante du carpe diem, achève de faire de ce poète un dramaturge progressiste à la philosophie épicurienne, contrairement au Gringoire de Hugo, modéré, opportuniste, vaniteux, dont la "très-belle moralité ", allégorique elle aussi, mais de circonstance, avait pour titre Le Bon Jugement de madame la Vierge Marie, et se caractérisait par une pléthore d' "incommensurables tirades" et de personnages "empaillés », peu susceptibles de lui attirer la sympathie de l'auteur de la préface de Cromwell. Tout se passe comme si Dieterle et ses scénaristes avaient décidé de prendre au pied de la lettre les éloges et la compassion ironiques du romancier à l'égard de son personnage et de faire de Gringoire le héros de l'histoire. De là à en faire le héraut de la liberté de pensée via l'imprimerie (« ceci ») contre le dogmatisme de l'Église (« cela»), il n'y aurait eu qu'un pas, si un tel développement, actualisant les paroles de l'archidiacre - que Hugo interprétait comme «le cri du prophète [...] qui voit dans l'avenir l'intelligence saper la foi, l'opinion détrôner la croyance ${ }^{20} »-$, n'avait été rendu impossible par le code Hays. Les scénaristes avaient bien trouvé leur héros positif mais ils se voyaient contraints de ne pas l'opposer à l'institution - l'Église - et aux valeurs la foi, la croyance -, mises en question, d'après Hugo, par l'imprimerie. Il s'agissait donc de lui trouver d'autres adversaires et de déplacer quelque peu le débat.

La référence au " peuple » dans le texte du carton liminaire ne suggérait-elle pas que le conflit idéologique allait se doubler d'une lutte des classes, voire se confondre avec elle? Cette orientation n'est pas absente du scénario mais, comme on s'en doute, ne pouvait guère être poussée très loin dans un pays où toute trace de marxisme était suspecte. Contrairement à ce qui se passe dans le roman, lors de la condamnation de Quasimodo à être fouetté et exposé au pilori, des voix critiques se font entendre parmi les spectateurs; l'une d'elles avance que «si tous les nobles recevaient ce qu'ils méritaient, on n'aurait pas assez de piloris ». Clopin $^{21}$ (précédemment identifié dans le cadre de la Cour des Miracles comme roi des mendiants) ayant révélé que les agissements de ses sujets étaient couverts par la protection achetée de la noblesse, Gringoire reconnaît ironiquement en cette dernière la gardienne de «l'ordre ancien et sacré » dont Frollo s'était aussi, dans le prologue, institué protecteur. La noblesse détenant elle-même ses privilèges du roi, une liaison d'intérêts se dessine du sommet 
de l'État jusqu'aux bas-fonds de la société. Mais cette situation a une origine: Clopin rappelle que « des milliers d'entre nous, après la guerre [de Cent Ans, précise-t-il, mais cela ne vaudrait-il pas pour celle de 1914-1918 ?], allaient de porte en porte demandant du travail honnête ». Et ils furent punis pour mendicité. «La classe dominante n'a pas dit "Travaille ou meurs de faim", elle a dit "Meurs de faim, car tu n'auras pas de travail". » D'où l'organisation de la " guilde des Mendiants ", qui se situe entre syndicat de chômeurs et syndicat du crime - car on y a recours au vol mais, plaide Clopin, à une bien moins grande échelle que la noblesse qui pratique «le pillage en masse d'une nation ». Les scénaristes auraient-ils trouvé la cible recherchée ? Oui et non, car Clopin et Gringoire songent bien à écrire un jour un livre sur la vérité de la misère, mais non à s'attaquer directement à la noblesse.

9 La lutte qui va s'engager pourrait nous ramener à l'enjeu soulevé par Hugo. Pour remédier au surpeuplement des prisons, pleines, non de voleurs ou de meurtriers de droit commun, mais "d'hérétiques, de libres-penseurs et d'orateurs séditieux », Frollo se déclare partisan d'augmenter non les prisons mais les exécutions. Après qu'il a poignardé Phœbus, il laisse accuser Esmeralda du meurtre, sous prétexte qu'elle l'aurait ensorcelé. Gringoire essaie de faire imprimer un appel aux Parisiens : "Jour après jour, des gens innocents disparaissent. Certains meurent au gibet, d'autres sont enterrés vivants dans des donjons"; maître Fischer lui promet les cent premiers exemplaires pour le lendemain, Gringoire prévoit de les faire distribuer par les étudiants et les mendiants de la Cour des Miracles, mais les presses sont détruites sur ordre de Frollo comme invention diabolique. "Vous pouvez détruire la matière, mais pas l'esprit", s'écrie Gringoire. Au tribunal, il tente de soulever l'auditoire en l'avertissant que nul n'est à l'abri du sort réservé à l'innocente Esmeralda. L'imprimerie retrouve ainsi les ennemis dont Hugo, commentant la prophétie terrifiée de Frollo, prédisait qu'elle triompherait: ceux qui préconisent les arrestations arbitraires et la persécution pour des motifs religieux et politiques.

Mais les scénaristes de Dieterle se gardent bien de donner à Louis XI l'initiative de la violation du droit d'asile, que le roi légitime, dans le roman, par d'augustes précédents $^{22}$. Le projet en est attribué, dans le film, à des nobles, qui comptent sur la signature du « comte Frollo » au bas de leur pétition pour faire pression sur le roi. Face à eux, deux stratégies se déploient : celle de Gringoire - bien éloigné de l'humble poète $\mathrm{du}$ roman, plaidant pour sa propre vie devant Louis $\mathrm{XI}^{23}$ - qui s'appuie hardiment sur l'imprimerie pour en appeler au roi et au peuple, celle de Clopin et de la Cour des Miracles, fondée sur la force. Le pamphlet de Gringoire, distribué par artisans et étudiants, se retrouve entre les mains du roi; celui-ci s'en fait lire le contenu par Frollo : « Le peuple a confiance en son roi, et exprime sa certitude, aussi longtemps que les cours de justice useront de la torture au lieu de bon sens, qu'il refusera la demande des nobles de désanctuariser Notre-Dame "; une manifestation sous les fenêtres du roi appuie la pétition ; Louis XI, faisant preuve de sa clairvoyance habituelle dans le film de Dieterle, discerne aussitôt que «cette nouvelle façon audacieuse de revendiquer par l'intermédiaire d'une pétition imprimée est en passe de créer une sorte d'opinion publique qui est capable de forcer dans leurs décisions même les rois ». " Impertinent, ajoute-t-il, mais j'aime ça ; c'est différent. » Il ne se fâche que lorsque l'archevêque lui apprend que des milliers de mendiants sont en train de prendre d'assaut Notre-Dame. Mais contrairement au Louis XI du roman, uniquement soucieux de préserver son autorité royale, il est parfaitement informé de leurs motivations et, Frollo ayant avoué le meurtre de Phœbus, il ne désire qu'arrêter le coupable, sauver l'innocente et 
défendre la cathédrale. Tel est aussi l'objectif des artisans parisiens mobilisés par le pamphlet de Gringoire pour la défense du droit d'asile et auxquels se joint le clergé pour barrer les portes de Notre-Dame. Clopin, ne croyant pas à leur détermination de s'opposer aux nobles, lance ses troupes à l'attaque. Écrasés et ébouillantés par Quasimodo, massacrés par les troupes du roi, ils paieront cher ce choix de la violence. Quasimodo triomphe et, dans son rugissement de victoire, on devine le mot « alléluia » (louez Dieu), que l'on retrouvera chanté en accompagnement du travelling arrière aboutissant à un plan d'ensemble de Notre-Dame, par lequel s'achève le film.

11 L'heureux dénouement, qui voit Esmeralda sauvée et unie à Gringoire, s'éloigne considérablement de celui de Hugo, où elle se retrouvait livrée au bourreau par Frollo et où Quasimodo ne s'unissait qu'à une morte, mais il pourrait passer pour une démonstration, inspirée par Hugo, que l'imprimerie est en mesure de faire échec à l'arbitraire et à la superstition; que la presse crée une opinion publique capable d'imposer ses décisions même aux rois ; que point n'est besoin de la force ; qu'il faut avoir confiance dans la puissance de la pensée et de la parole imprimée et largement diffusée ; bref, que « ceci »- l'imprimerie, la presse, l'intelligence, l'opinion publique a tué « cela »- les préjugés, la justice dévoyée par les intérêts personnels. À condition de négliger le fait que Hugo situe ces heureuses conséquences de l'invention de l'imprimerie dans le futur, et non dans l'époque de l'intrigue, et que si, dans le film même, les idées rétrogrades et l'abus de pouvoir sont bien terrassés en la personne de Frollo, ce n'est pas seulement grâce à l'imprimerie... Il faut un Quasimodo pour poser le pied sur le poignard dont veut encore s'emparer le Grand Juge, puis pour jeter son maître par-dessus la balustrade. Et ce n'est pas à l'imprimerie seule qu'Esmeralda et les Gitans doivent leur salut. Il faut, pour l'assurer, la bienveillance royale et archiépiscopale, le concours du trône et de l'autel. Gringoire le proclame bien aux Parisiens : « Nous avons gagné ! le roi l'a graciée. » L'archevêque le confirme : « Elle est graciée. Son peuple peut vivre libre partout en France. » À Esmeralda qui lui baise respectueusement la main il a beau déclarer: "Ne me remercie pas, remercie Quasimodo, qui t'a sauvée de la pendaison, et Gringoire, dont la petite brochure imprimée t'a libérée ", Gringoire lui-même ne se jette pas dans les bras d'Esmeralda sans prendre la main de l'archevêque qu'il salue avec reconnaissance d'un «Votre Grâce ».

12 C'est peu de dire que, dans le film, la presse à imprimer n'a pas tué l'Église; elle a contribué à la sauver et même à la renforcer. Sans doute le code Hays y est-il pour quelque chose, lui qui interdisait de critiquer l'Église et la foi. Mais fallait-il pousser le respect des grands hommes de l'histoire jusqu'à faire de Louis XI le porte-parole de Hugo? Hugo qui considérait, précisément dans le chapitre "Ceci tuera cela", l'invention de l'imprimerie comme "la révolution-mère ", et laissait entendre, en une autre circonstance du roman, que "l'heure du peuple» n'était pas venue mais qu'elle sonnerait le jour où la Bastille croulerait ${ }^{24}$. Perspective révolutionnaire que le film semble implicitement refuser, en faisant reconnaître à Clopin lui-même, mourant, son erreur : avoir cru que sauver Esmeralda sans recours à la force n'était qu'un rêve de poète.

13 L'espoir annoncé par le carton initial n'était-il pour le peuple qu'un rêve ? On pourrait se le demander, à entendre Quasimodo, en haut d'une tour de Notre-Dame, interroger une gargouille sur l'épaule de laquelle il appuie sa tête: «Pourquoi n'ai-je pas été fait de pierre comme toi ${ }^{25}$ ?». Le préjugé qui tend à exclure un être physiquement difforme 
du bonheur amoureux et à le vouer à la solitude a l'air tenace. Cette ultime note d'amertume paraît néanmoins submergée par l'alléluia final.

\section{Medak dans le sillage de Dieterle, mais dans le contexte de l'intégrisme et délivré du code Hays}

Plus d'un demi-siècle après, Peter Medak et son scénariste, John Fasano, semblent bien vouloir se démarquer sensiblement du film de Dieterle en situant l'action, dès le carton initial, en 1480, époque définie comme "ténébreuse et effrayante » et en mettant en cause l'Église et non plus seulement la superstition: "C'était un monde où les idées nouvelles étaient proscrites par l'Église et où la simple possession d'une page imprimée était un crime passible de la peine de mort ${ }^{26}$. » Avertissement illustré aussitôt par la saisie, sous la direction d'un Claude Frollo en tenue d'ecclésiastique, d'une presse à imprimer, qu'il va désigner comme "l'instrument de Satan ». Le prêtre fanatique semble préfigurer ici quelque ayatollah ou mollah. La prise de Kaboul par les talibans date précisément de l'année où se tourne le film : 1996.

Après cette séquence choc, nouveau carton : vingt-cinq ans plus tard - en 1505 ? -, le jour de la fête des Fous. Anachronisme des décorateurs : une des tours de Notre-Dame est en cours de construction, alors qu'elles sont achevées depuis plus de deux siècles et demi. Mais passons. Va-t-on assister au mystère de Gringoire tel que Hugo le présente ? Point du tout. Gringoire, plus jeune premier encore ${ }^{27}$ que chez Dieterle, harangue la foule et distribue même des sortes de tracts: «La cour d'Espagne, proclame-t-il, a envoyé un homme à travers les océans pour découvrir un nouveau monde mais notre roi, notre roi voudrait nous faire admettre qu'il n'y a pas de monde par-delà les murs de cette cité!» S'agirait-il d'une critique implicite de l'idéalisation du roi dans la version de Dieterle, sur un sujet où précisément celle-ci lui faisait crédit d'une particulière clairvoyance? Le discours de Gringoire unit ensuite dans la même réprobation les deux pouvoirs, clérical et monarchique :

«Pourquoi n'y a-t-il pas de livres dans Paris à l'exception de ceux de nos églises?

[...] Parce que l'Église et l'État partagent la même opinion [...], à savoir que vous ne devez avoir aucune opinion personnelle! Habitants de Paris, le savoir, c'est le pouvoir! Ne restons pas assis à ne rien faire! Révoltons-nous! Soulevons-nous! Tous debout !... ».

Plus d'allégorie, ni conformiste ni progressiste, un appel politique direct à l'insurrection. On ne peut être plus clair. Mais « ceci » tuera-t-il « cela »?

L'adaptation se rapproche singulièrement de celle de 1939 pour l'élection du « roi des fous ", à cette différence près qu'intervient un nouveau personnage, qualifié de "premier ministre » et nommé Gauchère ${ }^{28}$. Apprenant au roi que "l'archidiacre Frollo fait rechercher par la garde royale tous les livres qui sont dans Paris ", il demande «quel danger potentiel » les livres dissimulent. À quoi le souverain ${ }^{29}$ répond: «Dom Frollo est âgé, il est un peu maniaque à vrai dire. » Façon de s'en distinguer.

À la Cour des Miracles, Clopin montre plus d'agressivité envers Gringoire que chez Dieterle: «Tu veux nous aider, prendre publiquement notre défense! Mais qu'est-ce que tu sais de nous, hein? Tu es encore pire que les nobles! Tu... tu es un... un intellectuel! Eux, ils nous traitent comme du bétail et toi, tu fais de nous l'une de tes grandes causes! Ce n'est pas de cela dont tu garniras notre table! Tu es entré dans le royaume des pauvres, des opprimés, sans avoir été opprimé le moins du monde !» 
19 Il faut attendre que soit passée la scène du pilori pour assister à l'équivalent de celle que le film de 1939 s'était donnée pour prologue et qui, dans le roman, précède le dialogue de sourds entre le juge et Quasimodo. Dans le scénario bâti par John Fasano, le roi se rend à Notre-Dame en compagnie de son premier ministre, et y est reçu par un Frollo $^{30}$ qu'il appelle "Votre Éminence ». Le monarque note lui-même que dix ans se sont écoulés depuis son couronnement, détail qui, recoupé avec l'allusion à la recherche en cours d'un nouveau monde par une expédition espagnole (et peut-être avec les dates approximatives de naissance et de mort du vrai Gringoire : 1475-1538) semble indiquer que le roi de France n'est plus, dans cette adaptation, Louis XI, monté sur le trône en 1461 et mort en 1483. Cette fois, l'initiative de la visite est attribuée au premier ministre, qui tient la place du médecin du roi chez Hugo et qui présente l'invention de Gutenberg. Frollo s'en défie à peu près dans les mêmes termes que son modèle romanesque et surtout que son prédécesseur chez Dieterle comme d'une machine infernale qui peut «renverser un royaume». Face à lui, le roi adopte à peu près la position du Louis XI de Dieterle.

20 Le livre imprimé dont ils parlent ne s'intitule plus De la liberté de penser mais Liberté, ce qui n'est guère moins anachronique, d'autant qu'il s'agit d'un «appel à la liberté ». L'auteur n'en est plus Gringoire comme dans la version de 1939, mais un inconnu: «Avec la presse, déclare Frollo, tout le monde peut devenir un auteur, et les opinions de n'importe qui deviennent aussi importantes que celles d'un autre.» La discussion aborde ensuite le point de savoir si ce que Frollo refuse, ce sont les pamphlets politiques ou la diffusion de la connaissance. Gauchère hérite de l'esprit d'ouverture de Louis XI chez Dieterle : «L'imprimerie peut répandre le savoir dans les masses avec plus de facilité.» L'archidiacre réplique que cette facilité d'acquisition de la connaissance la dévalorisera : « Tout le monde aura des livres et ces livres n'auront plus aucune valeur."

21 Le roi n'a guère à lui répondre et se contente de reprendre textuellement l'opposition entre les cathédrales, " écriture du passé », et la presse, "écriture de notre époque », ainsi que sa détermination à ne rien faire " pour arrêter son développement ». Frollo se réfère alors à une promesse du père du souverain - Charles VIII ? - de "soutenir l'Église dans sa proscription des ouvrages imprimés ». Et, avant qu'il ne réitère la volonté que lui prêtaient les scénaristes de Dieterle de protéger la France des livres imprimés " comme des sorcières, des envoûteurs et des bohémiennes ", c'est le premier ministre qui lui donne une cinglante réplique, dans des termes - « Je ferai entrer ce pays dans le seizième siècle » - qui semblent suggérer que c'est là un spécimen de la perpétuelle querelle des Anciens et des Modernes et qu'il suffirait de remplacer «seizième " par "vingt-et-unième » siècle pour en sentir la permanente actualité. Un peu plus tard, Frollo lui rendant sa visite, Gauchère continue à s'exprimer comme un premier ministre d'aujourd'hui : «Le règne de Louis XII sera le règne du changement »; «le Roi est partisan du changement. " Le doute se lève ainsi peu à peu sur l'identité du monarque : non Louis XI, comme le laisse encore entendre la version française (sans doute pour se conformer au roman), mais Louis XII.

Gauchère est surpris en train de lire un livre de Marsilio Ficino que Frollo désigne aussitôt comme "un humaniste italien ». Cette référence, non tirée du roman, au traducteur florentin des dialogues platoniciens Marsile Ficin (1433-1499) peut surprendre: on croyait le scénariste de Medak bien indifférent à toute exactitude historique et voilà la trace d'une documentation. Le dialogue se resserre : «Le monde 
que vous cherchez à protéger sera bientôt dépassé ", affirme Gauchère. « Le monde que vous cherchez à établir, vous ne le verrez jamais ", réplique Frollo. Et pour cause : après avoir embrassé le premier ministre sur le front, il lui plante dans le ventre le couteau d'Esmeralda. Meurtre politique, donc, et non plus passionnel.

Mais le lecteur du roman n'est pas au bout de ses surprises. Quasimodo, après avoir arraché Esméralda au gibet, lui fait des confidences inédites : « Mes parents à moi, je ne les ai pas connus non plus. Ils n'étaient pas aveugles mais juste incapables... de m'aimer. Ils m'ont déposé sur les marches de la cathédrale, croyant que j'allais y mourir.» Rappelons que, dans le roman, ce sont des « Égyptiens » qui l'ont déposé chez Paquette à la place de la fille de celle-ci, Agnès, et que c'est l'archevêque de Reims qui l'a envoyé à Paris pour être exposé comme "enfant trouvé ${ }^{31}$ ». Mais le plus fort reste à venir : les livres qui sont conservés dans le sous-sol de la cathédrale sont devenus ses seules fréquentations ; il les a tous lus, l'un après l'autre, et voici le résultat :

«Grâce à leur compagnie, aujourd'hui j'ai de nombreux amis. Ils vont de Cicéron jusqu'aux Pères célestins. Ils m'ont appris la scolastique, la poétique, [...] et même l'hermétique, cette sagesse plus haute, il me semble, que toutes les autres. Pour... pour tout vous dire, je... j'écris un livre. Il va comporter au moins six cents pages quand je l'aurai terminé. »

Comme Esmeralda n'a " encore jamais vu de livre », il lui en apporte un, et comme elle ne sait pas lire, il lui explique que c'est du latin et que ce sont Les Vies[parallèles] de Plutarque ; traduites du grec, faut-il supposer... Quasimodo, lecteur de Plutarque et grand érudit, voilà qui renouvelle notre connaissance du personnage! En fait, il hérite là de caractéristiques attachées dans le roman à Gringoire qui, au cours de sa « nuit de noces ", confiait à Esmeralda devoir à Frollo d'être devenu " un véritable lettré, sachant le latin depuis les Offices de Cicero jusqu'au Mortuologe des pères célestins; et n'étant barbare ni en scolastique, ni en poétique, ni en rythmique, ni même en hermétique, cette sophie des sophies"; Gringoire encore, auteur d'" un livre qui aura six cents pages sur la comète prodigieuse de $1465^{32}$ ». Seule donc la présence des Vies parallèles de Plutarque est une invention complète du scénariste.

Mais John Fasano ne se contente pas de transférer sur Quasimodo l'instruction reçue par le Gringoire du roman. Il lui attribue la rédaction du pamphlet que Dieterle et ses scénaristes avaient imaginé de faire écrire à Gringoire. Il améliore de plus la coordination de son action avec celle de la Cour des Miracles. Quasimodo s'y rend, tend un paquet à Gringoire. "Qu'est ce que c'est que ça?» interroge celui qui n'est plus désigné que comme "l'orateur ». C'est un appel « au peuple de Paris »: «Parisiens, jour après jour, des gens innocents disparaissent. Certains meurent au gibet, d'autres sont enterrés vivants dans des donjons. » On reconnaît là très exactement le texte de l'appel lancé par le Gringoire de Dieterle au moment du procès d'Esmeralda, dont maître Fisher lui promettait cent exemplaires pour le lendemain. Si l'on se souvient de cela, la question de Gringoire - «C'est vous qui avez écrit ça ?» - gagne encore en pertinence. Quasimodo confirme: «C'est moi! moi!» Autre progrès dans l'union des forces populaires : il n'y a plus de divergence de stratégie entre Gringoire et Clopin. À la scène suivante, on apprend de la bouche même du roi, moins compréhensif en la circonstance que le Louis XI de Dieterle, que les pamphlets sont «distribués par des brigands, des artisans et des étudiants dans tout Paris ». Frollo relève que c'est "le produit d'une presse à imprimer ». Étonnement du roi : il y en a donc une qui a échappé à la vigilance de Frollo! Réponse de Son Éminence : «Nobody is perfect. » Ou plus exactement: «Yes, yes, I am not perfect. " S'écartant totalement et du Louis XI du roman et de celui de 
l'histoire, le roi semble prendre le parti de ses "amis les nobles", s'associer à la proscription des livres imprimés et des presses, préconisée par un Frollo qui paraît investi du pouvoir de réprimer les responsables du pamphlet. Tout se passe comme si, après l'assassinat, attribué à Esmeralda, d'un premier ministre libéral et progressiste, le pouvoir ou au moins l'influence était passée au partisan d'une ligne dure et réactionnaire. Le roi prend conseil de Frollo sur la conduite à adopter à l'égard de la bohémienne. Celui-ci s'en charge, en même temps qu'il décharge le monarque de sa responsabilité dans la violation du droit d'asile.

Mais le pamphlet fait son chemin et l'on assiste à une véritable manifestation dans le style de celles du XX siècle. Phœbus, dont le rôle se limite strictement à la direction des forces de l'ordre, fait son rapport au roi : «Des mendiants vont attaquer le Palais ! Ils sont dans la rue par centaines! » Le roi s'étonne que le peuple ose se révolter contre le verdict. Clopin et les siens arrivent alors en scandant « Esmeralda! Esmeralda! » et en distribuant des tracts. Gringoire s'élance et délie la corde du cou d'Esmeralda, ce qui lui vaut les acclamations de la foule, scandant le nom de la jeune bohémienne. De son côté, Clopin interpelle non plus, comme dans le roman ou chez Dieterle, "l'évêque de Paris » mais le monarque, identifié désormais sans équivoque possible (alors qu'il est interpellé sous le nom de Louis XI dans la version française) : «Je vous ordonne, roi Louis XII, de libérer la bohémienne. Le peuple de Paris est venu la sauver de tous ces nobles qui veulent la pendre. - [...] Qui êtes-vous ? demande le roi, interloqué. - Je suis Clopin! Le Roi... de... Paris. » Cela tournerait à la révolution si Gringoire, reconnaissant l'autorité du roi, ne venait plaider auprès de lui la cause d'Esmeralda.

Pour dénouer la situation, il faut trouver le meurtrier. Chez Dieterle, le bon archevêque Claude Frollo obtenait des aveux presque spontanés de son frère Jehan. Ici, le procédé est plus violent : Quasimodo suspend tout simplement Frollo dans le vide et le somme d'avouer; il lâche une de ses deux mains puis l'avertit qu'il mourra sans absolution de ses péchés. Soumis à cette double pression, Frollo finit par craquer et avouer; Esmeralda confirme; le roi est convaincu et pardonne. "Parisiens! elle est libre!" annonce Gringoire à la foule. Frollo demande son pardon à Quasimodo qui l'accorde. Tout est donc bien qui finit bien? Non! Péripétie : Frollo est irrécupérable, il veut encore s'attaquer à Esmeralda. Quasimodo s'interpose et s'embroche sur le couteau; Frollo ne l'a pas voulu mais se rue de nouveau sur Esmeralda, comme s'il la rendait responsable de la blessure de Quasimodo. Celui-ci bondit et entraîne Frollo dans l'abîme. Le prêtre s'écrase au sol, tandis que Quasimodo est hissé par Esmeralda aidée de Gringoire mais meurt de sa blessure, après avoir levé les yeux vers une statue et posé la même question que chez Dieterle: «Pourquoi n'ai-je pas été fait de pierre comme toi ?" Fin tout de même moins sinistre que celle du roman, où Esmeralda meurt, mais plus sombre donc que celle de Dieterle.

Quoi qu'il en soit, Medak donne ainsi au « ceci tuera cela » de Frollo une interprétation que le code Hays empêchait peut-être en partie Dieterle de formuler aussi radicalement, mais vers laquelle il tendait déjà. Cette interprétation est proche de la première que dégageait Hugo lui-même: «La presse tuera l'église. » Chez Dieterle, l'imprimerie contribue au rétablissement de la justice et précipite la mort du mauvais juge, substitut peut-être du mauvais prêtre ; chez Medak, elle permet d'obtenir justice contre l'Église, incarnée dans un prêtre dont la férocité n'a pas le contrepoids de quelque figure idéale d'ecclésiastique bienveillant, tel l'archevêque du film de Dieterle. 


\section{L'écran tuera-t-il le livre?}

Dans les deux films on entend «bruire et fourmiller l'humanité » sur la voie de son émancipation, «l'opinion détrôner ", sinon « la croyance ${ }^{33}$ ", du moins la superstition, le dogmatisme et l'intolérance. On assiste, par l'intermédiaire de l'imprimerie, à la naissance d'une opinion publique susceptible, surtout chez Medak, de détruire un royaume. Les deux films s'arrêtent au seuil de cette ultime conséquence politique de l'invention de l'imprimerie. Sans doute à cause de l'anachronisme et de la contre-vérité historique que constituerait le renversement du roi. Mais ils vont assez loin dans ce sens et même trop; non par audace, car Hugo fait aussi entrevoir dans Notre-Dame de Paris la chute de la Bastille et de la royauté ${ }^{34}$, mais par un excessif optimisme qui se communique à l'intrigue : ce que Hugo présente comme des prophéties se réalise, ou presque, sous nos yeux.

Ces deux films illustrent aussi d'une autre façon non prévue par Hugo le «Ceci tuera cela ». Par le processus d'adaptation, par leurs écarts, ils altèrent sensiblement le roman dont ils tirent leur substance. Et l'on s'aperçoit que ce qu'adapte le second, ce n'est pas seulement le roman de Hugo mais également, et peut-être surtout, le film de Dieterle. Telle a été aussi la démarche des scénaristes du dessin animé des studios Disney, que Roland Recht a évoqué. De médiation en médiation, l'adaptation ne risquet-elle pas de tuer ou de faire disparaitre l'œuvre adaptée ? Faut-il voir dans la sortie, en 2002, du Bossu de Notre-Dame II, nouvelle production des studios Disney, la preuve du caractère destructeur d'un média dévorateur, ou se réjouir des adaptations comme d'autant de signes de l'accès du roman d'Hugo au statut de mythe ? En tout cas, que l'on ne se contente pas de jugements expéditifs sur des objets de consommation culturelle aussi répandus que les adaptations! Il peut être du plus vif intérêt pour le connaisseur d'une œuvre de comparer ces adaptations à sa propre «lecture ", de les situer et de les analyser. Il est presque de son devoir d'en rendre compte.

\section{NOTES}

1. . Voir la filmographie de Notre-Dame de Paris dans Delphine Gleizes (dir.), L'œuvre de Victor Hugo à l'écran: des rayons et des ombres, Paris, L'Harmattan, Saint-Nicolas, Les Presses de l'université Laval, p. 252-256.

2. . Production RKO, avec Charles Laughton (Quasimodo) et Maureen O'Hara (Esmeralda).

3. . Production Alliance Communications (firme canadienne), avec Mandy Patinkin (Quasimodo) et Salma Hayek (Esmeralda).

4. . Toutes les citations d'extraits du film de Dieterle sont des traductions personnelles, aussi littérales que possible.

5. . Le code Hays est traduit d'après le texte qu'en donne le site web artsreformation.com (consulté le 10 mars 2010), http://www.artsreformation.com/a001/hays-code.html.

6. . Cedric Hardwicke interprète le Grand Juge, Walter Hampden l'Archevêque. La version doublée en français intervertit les prénoms, rendant au Grand Juge le prénom de l'archidiacre. 
7. . Livre V, 1, p. 279 de l'édition de Notre-Dame de Paris, introduction, notes et chronologie par Jacques Seebacher, Paris, Librairie générale française ("Le livre de poche classique »), 1998, qui sera notre édition de référence pour les citations du roman.

8. . Livre V, 2, p. 280.

9. . Ibid., p. 280, 283.

10. . Interprété par Harry Davenport.

11. . Livre V, 1, p. 267-268.

12. . Voir ibid., p. 278-279.

13. . Ibid., p. 267.

14. . Ibid., 2, p. 280.

15. . Ibid., p. 281, 297.

16. Ibid., p. 284.

17. . Auteur d'un Juarez, il sera chassé de Hollywood en tant que "premature antifascist » (antifasciste prématuré), et verra son passeport révoqué en 1951 et 1953.

18. . Je tiens cette précision, comme les indications de la note précédente, de Francis Mickus, auteur d'un mémoire de maîtrise sur le film de Dieterle soutenu à l'université Paris 3 en 1998.

19. . Interprété par Edmond O’Brien.

20. . Livre V, 2, p. 280.

21. . Interprété par Thomas Mitchell.

22. . Voir livre X, 5, p. 622-623.

23. . Voir ibid., p. 609-611.

24. . Voir ibid., p. 618-619.

25. . Confidence faite par Quasimodo, dans le roman, à un personnage grotesque sculpté : «Oh! que ne suis-je de pierre comme toi ! ", et surprise par Esmeralda (voir livre IX, 4, p. 525).

26. . Toutes les citations d'extraits du film de Medak sont tirées de sa version française sauf lorsque celle-ci s'écarte de la version originale; dans ces cas, je lui substitue des traductions personnelles, aussi littérales que possible.

27. Interprété par Edward Atterton.

28. Interprété par Nicholas Grace. Le nom de Gauchère est emprunté à celui d'une vieille femme, témoin, dans le roman (livre IV, 1, p. 234), de l'adoption de Quasimodo par Frollo.

29. . Interprété par Nigel Terry.

30. . Interprété par Richard Harris.

31. . Voir livre VI, 3, p. 333, 336-337.

32. . Livre II, 7, p. 186.

33. . Voir livre V, 2, p. 280.

34. . Voir livre X, 5, p. 618-620.

\section{RÉSUMÉS}

Dans le contexte de 1939, tenus de se conformer aux impératifs du code Hays, les scénaristes de Dieterle ont changé l'archidiacre Frollo en un «Grand Juge » raciste, Louis XI en un souverain éclairé, et Gringoire en héros positif, opposé non à l'Église, mise en question, d'après Hugo, par l'imprimerie, mais s'appuyant sur celle-ci pour en appeler au roi et au peuple. En 1997, Medak et son scénariste font de Frollo un prêtre fanatique qui semble préfigurer quelque ayatollah. Un 
«premier ministre », Gauchère, hérite de l'esprit d'ouverture du Louis XI de Dieterle. Après son assassinat par Frollo, la ligne réactionnaire l'emporte. Une manifestation tournerait à la révolution si Gringoire, d'abord hostile aux pouvoirs clérical et monarchique, ne reconnaissait l'autorité du roi. Les heureux dénouements des deux films pourraient passer pour la démonstration que, sans recours à la violence mise en œuvre par Clopin et la Cour des Miracles, « ceci » - l'imprimerie, l'intelligence, l'opinion publique - a tué « cela » - les préjugés, la justice dévoyée et, chez Medak, le dogmatisme et l'intolérance. Ils se montrent donc sensiblement plus optimistes que le roman. Ils illustrent aussi d'une autre façon, non prévue par Hugo, le «Ceci tuera cela ». Par leurs écarts, les films altèrent sensiblement le roman. Et ce qu'adapte Medak, ce n'est pas seulement Hugo mais également, et peut-être surtout, Dieterle. De médiation en médiation, l'adaptation ne risque-t-elle pas de tuer l'œuvre adaptée?

In the context of 1939, and obliged to conform to the requirements of the Hays Code, Dieterle's scriptwriters changed the archdeacon Frollo into a racist "Great Judge", Louis XI into an enlightened monarch. They made Gringoire into a positive hero, someone not opposed to the Church - which according to Hugo is challenged by the advent of printing - but rather someone who in fact relies on printing in order to appeal to the king and the people. In 1997, Medak and his scriptwriter turn Frollo into a fanatical priest who seems to prefigure some ayatollah. A "prime minister", Gauchère, inherits the open-mindedness of Dieterle's Louis XI. After Frollo kills him, reactionary forces take advantage to gain control. A demonstration would turn into revolution if Gringoire, at first hostile to the powers of the clergy and monarchy, did not finally recognize the king's authority. The happy endings of the two films could be taken to show that, without the recourse to violence by Clopin and his Court of Miracles, "this" - printing, intelligence, public opinion - killed "that" - prejudice, warped justice, and, for Medak, dogmatism and intolerance. Thus these films appear considerably more optimistic than the novel. They also illustrate "This will kill that" in another way that Hugo did not anticipate. Through their divergences, they noticeably alter the novel. And the second film adapts not only Hugo but equally - and, perhaps, mostly - Dieterle. From alteration to alteration, doesn't the adaptation risk killing the adapted work?

In dem Kontext des Jahres 1939 haben Dieterles Drehbuchschreiber, die sich an die zwingenden Vorschriften des Code Hays halten mussten, den Erzdiakon Frollo in einen rassistischen Hauptrichter, den König Ludwig XI. in einen aufgeklärten Monarchen verwandelt. Aus Gringoire wurde ein positiver Held: er widersetzte sich gar nicht der Kirche , die nach Victor Hugo vom Buchdruck in Frage gestellt worden war, im Gegenteil stützte er sich auf sie, um an den König und an das Volk zu appellieren. 1997 machten Medak und sein Drehbuchschreiber aus Frollo einen Ayatollah ähnlichen fanatischen Priester. Ein Premierminister, Gauchère, erbte von der Klarsicht von Dieterles König Ludwig XI. Nach dessen Ermordung durch Frollo siegt die rückschrittliche Linie: Eine Kundgebung würde sich in eine Revolution umwandeln, wenn Gringoire, der sich ursprünglich gegen die kirchlichen und königlichen Mächte entschieden hatte, schließlich die Autorität des Königs nicht anerkennen würde. Die glücklichen Enden der beiden Filme könnten davon zeugen, dass ohne die Anwendung von Gewalt durch Clopin und seinen Hof der Wunder, « dieses »- Buchdruck, Klugheit, öffentliche Meinung - « jenes »Vorurteile, lasterhafte Justiz, und bei Medak Dogmatismus und Intoleranz - getötet hat. Sie bieten eine andere Auffassung des " Dieses wird jenes töten ", an welche Hugo nicht gedacht hatte. Die Filme verfälschen augenscheinlich den Roman. Medak verfilmt nicht nur Hugos Werk, sondern auch, und vielleicht hauptsächlich, Dieterles. Ob so von Veränderung zu Veränderung die Adaptation nicht riskiert, das adaptierte Werk selber zu töten? 


\section{AUTEUR}

\section{ARNAUD LASTER}

Maître de conférences de littérature française à la Sorbonne nouvelle, Arnaud Laster a porté ses recherches sur les œuvres de Hugo et de Prévert et notamment sur les rapports de la musique, de l'opéra et du cinéma avec leurs textes. Il a participé aux éditions des Cuvres complètes de Hugo (Le Club français du livre et « Bouquins », Robert Laffont) et procuré, avec Danièle Gasiglia, celle des cuuvres complètes de Prévert («Bibliothèque de la Pléiade », Gallimard, 2 vol.). Auteur de Pleins feux sur Victor Hugo (Comédie-Française), de la préface et du dossier des Misérables (Pocket), il a édité La Légende des siècles (« Poésie », Gallimard) et Le Théâtre en liberté (« Folio », Gallimard), comparé « Victor Hugo et Berlioz face à la musique » (dans Victor Hugo et les images, Dijon), dirigé un $n^{\circ}$ Hugo de L'Avant-Scène Opéra. Il a mené une réflexion sur ce que pourrait être une édition critique des textes de Prévert pour le film Notre-Dame de Paris (Éditer des œuvres médiatiques, Québec), analysé son adaptation du roman (CinémAction, $\mathrm{n}^{\circ}$ Prévert), étudié « Le cinéma dans le cinéma, à propos des Amants de Vérone de Prévert et Cayatte »(dans Le Cinéma au cinéma, Luxembourg, Lansman), «Les représentations du théâtre de Hugo à la télévision » (dans L'œuvre de Victor Hugo à l'écran, Québec), posé la question : « Adapter, est-ce toujours altérer ? Les Misérables à l'écran » (Paris Musées). Il a été parmi les fondateurs de la Société des Amis de Victor Hugo et du festival Victor Hugo et égaux, qu'il préside. 